\title{
Stability with respect to the domain of a nonlinear variational inequality
}

\author{
Daniela Inoan
}




\title{
STABILITY WITH RESPECT TO THE DOMAIN OF A NONLINEAR VARIATIONAL INEQUALITY
}

\author{
DANIELA INOAN
}

[Received: August 22, 2002]

\begin{abstract}
In this paper we investigate the stability with respect to the domain perturbations of a class of nonlinear variational inequality.

Mathematics Subject Classification: Primary 47J20, 90C31; Secondary 49Q12
\end{abstract}

Keywords: parametric variational inequalities, mapping method

\section{INTRODUCTION}

The mathematical theory of shape optimization benefits of a vast literature; the optimal shape design for systems governed by PDEs was studied in a great many papers, see for example [1], [2], [3]. Also, problems where variational or hemivariational inequalities appear were treated in [1], [4], [5], [6] and others.

A problem of shape optimization for a system described by a variational inequality can be looked at as a problem of optimal control in which the role of the control is played by sets from a class of admissible domains and the variational inequality appears as the state equation. In this context it is interesting to study the behavior of solutions to the variational inequality when the domain is perturbed.

The purpose of this paper is to study the stability with respect to the domain perturbations of a nonlinear variational inequality of the form

Find $u_{\Omega} \in K(\Omega)$ such that

$$
\left\langle\mathcal{A}\left(\Omega, u_{\Omega}\right), v-u_{\Omega}\right\rangle \geq 0, \forall v \in K(\Omega),
$$

where $\Omega$ is a bounded open subset of $\mathbb{R}^{N}, K(\Omega)$ is a closed, convex set in the Sobolev space $H^{1}(\Omega)$ and $\mathcal{A}$ is a nonlinear operator of a special form, $\mathcal{A}(\Omega, u) \in\left(H^{1}(\Omega)\right)^{*}$. If $\Omega_{0}$ is fixed in the class of the admissible domains and $u_{0}$ is the solution of $(V I)_{\Omega_{0}}$, the following problem arises: Is there a neighborhood $V_{0}$ of $\Omega_{0}$ (in a sense that will be specified) and a mapping $\theta$ defined on this, continuous at $\Omega_{0}$, with $\theta\left(\Omega_{0}\right)=u_{0}$ and such that $\theta(\Omega)$ is a solution of $(V I)_{\Omega}$ for each $\Omega \in V_{0}$ ? 
General stability results for parametric variational inequalities under small perturbations of the parameter have been given in [7], [8]. One of these results is presented in Section 3 and used in the paper.

In order to define the topological space of the parameters as a space of functions we use the mapping method (Section 2) for which the basic results were established by Murat and Simon in [3].

Finally, in Section 4 we present the main result of the paper and an example for a linear variational inequality.

\section{The MAPPING METHOD}

The mapping method consists in defining the class of admissible domains as images of a fixed set. The main notions and properties were established in [3]; we present some of them following [4], [2].

Let $C \subset \mathbb{R}^{N}$ be a bounded, open set, with $\partial C$ of class $W^{i, \infty}, i \geq 1$ and such that $\operatorname{int} \bar{C}=C$.

We consider the following spaces:

$$
\begin{aligned}
& W^{k, \infty}\left(\mathbb{R}^{N}\right)^{N}=\left\{\phi \mid D^{\alpha} \phi \in L^{\infty}\left(\mathbb{R}^{N}\right)^{N} \forall \alpha \text { with } 0 \leq|\alpha| \leq k\right\} \\
& \mathcal{F}^{k, \infty}=\left\{S: \mathbb{R}^{N} \rightarrow \mathbb{R}^{N} \mid S \text { bijective, } S-I, S^{-1}-I \in W^{k, \infty}\left(\mathbb{R}^{N}\right)^{N}\right\} \\
& O^{k, \infty}=\left\{\Omega \mid \Omega=S(C), S \in F^{k, \infty}\right\}
\end{aligned}
$$

and the norm in $W^{k, \infty}\left(\mathbb{R}^{N}\right)^{N}$ :

$$
\|S\|_{k, \infty}=\operatorname{ess} \sup _{x \in \mathbb{R}^{N}}\left(\sum_{0 \leq|\alpha| \leq k}\left|D^{\alpha} S\right|_{N}^{2}\right)^{1 / 2}
$$

$\|\cdot\|_{N}$ represents the norm on $\mathbb{R}^{N}$. The norm on $L^{\infty}\left(\mathbb{R}^{N}\right)\left(\right.$ or $\left.L^{\infty}\left(\mathbb{R}^{N}\right)^{N^{2}}\right)$ will be denoted by $\|\cdot\|_{\infty}$.

$O^{k, \infty}$ consists in a family of bounded and open sets. If $\partial C$ is of class $W^{k, \infty}, k \geq 1$ then $\partial \Omega$ is of class $W^{k, \infty}$ also.

We define on $O^{k, \infty} \times O^{k, \infty}$ a function

$$
\delta_{k, \infty}\left(\Omega_{1}, \Omega_{2}\right)=\inf _{S \in \mathcal{F}^{k, \infty}, S\left(\Omega_{1}\right)=\Omega_{2}}\left(\|S-I\|_{k, \infty}+\left\|S^{-1}-I\right\|_{k, \infty}\right) .
$$

It can be proved (see [4], [2]) that there exists a positive constant $\mu_{k}$ such that

$$
d_{k, \infty}\left(\Omega_{1}, \Omega_{2}\right)=\left(\min \left\{\delta_{k, \infty}\left(\Omega_{1}, \Omega_{2}\right), \mu_{k}\right\}\right)^{1 / 2}
$$

is a complete metric on $O^{k, \infty}$.

Remark 1. It is known (see [3]) that $\Omega_{n} \rightarrow \Omega$ in $O^{k, \infty}$ if and only if there exist $S_{n}, S \in \mathcal{F}^{k, \infty}$ such that $\Omega_{n}=S_{n}(C), \Omega=S(C)$ and $S_{n} \rightarrow S, S_{n}^{-1} \rightarrow S^{-1}$ in $W^{k, \infty}\left(\mathbb{R}^{N}\right)^{N}$.

The next Lemma (see [4]) summarizes some properties used in the paper: 
Lemma 1. (a) If $S \in \mathcal{F}^{k, \infty}, \Omega=S(C)$, then $u \in H^{1}(\Omega)$ if and only if $u \circ S \in H^{1}(C)$. If $u_{n} \rightarrow u$ in $H^{1}(\Omega)\left(\right.$ or in $\left.H^{1}(C)\right)$ then $u_{n} \circ S \rightarrow u \circ S$ in $H^{1}(C)\left(\right.$ or $u_{n} \circ S^{-1} \rightarrow u \circ S^{-1}$ in $H^{1}(\Omega)$ ).

(b) Let $k \geq 1, u \in H^{1}\left(\mathbb{R}^{N}\right)$. The mapping $S \mapsto u \circ S$ is continuous from $\mathcal{F}^{k, \infty}$ to $H^{1}\left(\mathbb{R}^{N}\right)$.

(c) Let $k \geq 1$. The mappings $S \mapsto J S^{-1}$ and $S \mapsto \operatorname{det} J S$ are continuous from $\mathcal{F}^{k, \infty}$ to $W^{k-1, \infty}\left(\mathbb{R}^{N}\right)$ (with $J S$ we denoted the Jacobian matrix of $S$ ).

(d) If $u, v \in H^{1}(\Omega)$ and $\Omega=S(C)$ we have:

$$
\|u \circ S-v \circ S\|_{H^{1}(C)} \leq\left(\left\|\operatorname{det} J S^{-1}\right\|_{\infty}\right)^{1 / 2}\left(\|J S\|_{\infty}+1\right)\|u-v\|_{H^{1}(\Omega)} .
$$

\section{Parametric variational inequalities}

Let $H$ be a real, reflexive Banach space and denote with $H^{*}$ its dual. Let $W$ be a topological space, let $T: W \times H \rightarrow H^{*}$ and let $K: W \rightarrow 2^{H}$ be a set-valued map. For a given parameter $w \in W$ we consider the variational inequality:

$$
\begin{aligned}
(V I P)_{w} & \text { Find an element } x(w) \in K(w) \text { such that } \\
& \langle T(w, x(w)), y-x(w)\rangle \geq 0, \forall y \in K(w) .
\end{aligned}
$$

For a fixed $w_{0} \in W$ (the initial value of the parameter), suppose that $x_{0} \in K\left(w_{0}\right)$ is the solution of the corresponding problem $(V I P)_{w_{0}}$. We say that the problem $(V I P)_{w_{0}}$ is stable under perturbations if there exists a neighborhood $W_{0}$ of $w_{0}$ and a mapping $x: W_{0} \rightarrow H$, continuous at $w_{0}$, with $x\left(w_{0}\right)=x_{0}$ and such that, for each $w \in W_{0}, x(w)$ is a solution of $(V I P)_{w}$.

Definition 1. The map $T: W \times H \rightarrow H^{*}$ is called consistent in $w$ at $\left(w_{0}, x_{0}\right)$ if, for each $0<r \leq 1$, there exists a neighborhood $W_{r}$ of $w_{0}$ and a function $\beta: W_{r} \rightarrow \mathbb{R}$, continuous at $w_{0}$, with $\beta\left(w_{0}\right)=x_{0}$ such that for each $w \in W_{r}$, there exists $y_{w} \in K(w)$ such that

and

$$
\left\|y_{w}-x_{0}\right\| \leq \beta(w)
$$

$$
\left\langle T\left(w, y_{w}\right), z-y_{w}\right\rangle+\beta(w)\left\|z-y_{w}\right\| \geq 0,
$$

for each $z \in K(w)$ such that $r<\left\|z-y_{w}\right\| \leq 2$.

Definition 2. The maps $T(w, \cdot): H \rightarrow H^{*}$ are called uniformly strongly monotone on $W_{0} \subset W$ if there exists a positive constant $\alpha$ such that for all $w \in W_{0}$ and $x, y \in H$, $x \neq y$ we have :

$$
\langle T(w, x)-T(w, y), x-y\rangle \geq \alpha\|x-y\|^{2} .
$$

The following Theorem is a particular case of a Theorem proved in [7].

Theorem 1. In the above notations, let the set $K(w)$ be closed and convex for each $w \in W$. Consider $w_{0} \in W$ and $x_{0} \in K\left(w_{0}\right)$ fixed. Suppose that:

(i) $x_{0}$ is a solution of $(V I P)_{w_{0}}$;

(ii) $T$ is consistent in $w$ at $\left(w_{0}, x_{0}\right)$; 
(iii) there exists a neighborhood $V$ of $w_{0}$ such that the maps $T(w, \cdot)$ are uniformly strongly monotone, continuous from the line segments of $H$ to the weak topology of $H^{*}$, for all $w \in V$ and $x \in K(w)$.

Then the problem $(V I P)_{w_{0}}$ is stable under perturbations.

\section{Stability with ReSpect to the domain Of Perturbations}

Let $\Omega \subset \mathbb{R}^{N}$ be a bounded and open set. We consider the problem:

$(V I)_{\Omega} \quad$ Find $u_{\Omega} \in K(\Omega)$ such that

$$
\begin{aligned}
& \int_{\Omega} A\left(x, \nabla u_{\Omega}(x)\right) \cdot\left(\nabla v(x)-\nabla u_{\Omega}(x)\right) d x \\
& +\int_{\Omega} a\left(x, u_{\Omega}(x)\right)(v(x)-u(x)) d x \geq 0, \forall v \in K(\Omega)
\end{aligned}
$$

in the following notations and hypotheses:

$\left(\mathbf{H}_{\mathbf{1}}\right) K(\Omega) \subset H^{1}(\Omega)$ is a closed, convex, nonempty set,

$\left(\mathbf{H}_{2}\right) A=\left(a_{1}, \ldots, a_{N}\right)$ with $a_{j}: \mathbb{R}^{N} \times \mathbb{R}^{N} \rightarrow \mathbb{R}, a: \mathbb{R}^{N} \times \mathbb{R} \rightarrow \mathbb{R}$ having the properties:

(P1) $a_{j}(\cdot, \cdot), j=1, \ldots, N$ and $a(\cdot, \cdot)$ are measurable with respect to the first variable and continuous with respect to the second one,

(P2) $\left|a_{j}(x, \xi)\right| \leq c\left(k(x)+\|\xi\|_{N}\right)$ and $|a(x, \eta)| \leq c_{1}\left(k_{1}(x)+|\eta|\right)$ a.e. $x \in \mathbb{R}^{N}$, for all $\xi \in \mathbb{R}^{N}$, for all $\eta \in \mathbb{R}$, with $c, c_{1}$ positive constants and $k, k_{1}$ functions in $L^{2}(D)$ (for any bounded and open set $D$ ).

$$
\begin{aligned}
& \text { (P3) } \sum_{j=1}^{N} a_{j}(x, \xi) \xi_{j} \geq c_{2}\|\xi\|_{N}^{2}-c_{3} \text {, a.e. } x \in \mathbb{R}^{N} \text {, for all } \xi \in \mathbb{R}^{N}, \\
& \text { (P4) } \sum_{j=1}^{N}\left(a_{j}(x, \xi)-a_{j}(x, \tilde{\xi})\right)\left(\xi_{j}-\tilde{\xi}_{j}\right) \geq \gamma_{1}\|\xi-\tilde{\xi}\|_{N}^{2} \text {, a.e. } x \in \mathbb{R}^{N} \text {, for all } \xi, \tilde{\xi} \in \mathbb{R}^{N} \\
& \text { and }(a(x, \eta)-a(x, \tilde{\eta}))(\eta-\tilde{\eta}) \geq \gamma_{2}|\eta-\tilde{\eta}|^{2} \text {, a.e. } x \in \mathbb{R}^{N} \text {, for all } \eta, \tilde{\eta} \in \mathbb{R} \text {. }
\end{aligned}
$$

Theorem 2. In the conditions stated above, the variational inequality $(V I)_{\Omega}$ has at least one solution (see [9], p. 74).

Let $S \in \mathcal{F}^{k, \infty}$ such that $\Omega=S(C)$. Making the transform $x=S(X)$ in $(V I)_{\Omega}$ we get an equivalent problem on the fixed set $C$ :

Find $u_{S} \in K_{S} \subset H^{1}(C)$ such that

$$
\begin{aligned}
& \int_{C} A\left(S(X), J S^{-t}(X) \nabla u_{S}(X)\right) \cdot J S^{-t}(X)\left(\nabla v(X)-\nabla u_{S}(X)\right) \operatorname{det} J S(X) d X \\
& +\int_{C} a\left(S(X), u_{S}(X)\right)\left(v(X)-u_{S}(X)\right) \operatorname{det} J S(X) d x \geq 0, \quad \forall v \in K_{S},
\end{aligned}
$$

where $K_{S}=S K(\Omega)=\{u \circ S \mid u \in K(\Omega)\}$ is closed and convex. 
We define the operator $\mathcal{A}: \mathcal{F}^{k, \infty} \times H^{1}(C) \rightarrow\left(H^{1}(C)\right)^{*}$ as

$$
\begin{aligned}
\langle\mathcal{A}(S, u), v\rangle= & \int_{C} A\left(S(X), J S^{-t}(X) \nabla u(X)\right) \cdot J S^{-t}(X) \nabla v(X) \operatorname{det} J S(X) d X \\
& +\int_{C} a(S(X), u(X)) v(X) \operatorname{det} J S(X) d X, \forall v \in H^{1}(C) .
\end{aligned}
$$

Then the variational inequality can be written:

$(V I)_{S} \quad$ Find $u_{S} \in K_{S}$ such that $\left\langle\mathcal{A}\left(S, u_{S}\right), v-u_{S}\right\rangle \geq 0, \forall v \in K_{S}$.

We will apply Theorem 1 to this family of variational inequalities, considering $S \in$ $\mathcal{F}^{k, \infty}$ as the parameter.

Other hypotheses that we impose are:

$\left(\mathbf{H}_{3}\right)\left|a_{j}(x, \xi)-a_{j}(\tilde{x}, \tilde{\xi})\right| \leq \psi(x, \tilde{x})\left(\|\xi\|_{N}+\|\tilde{\xi}\|_{N}\right)+\phi(x, \tilde{x})\|\xi-\tilde{\xi}\|_{N}+\sigma(x, \tilde{x})$, for all $j=1, \ldots, N, x, \tilde{x}, \xi, \tilde{\xi} \in \mathbb{R}^{N}$; where $\psi(\cdot, \cdot), \phi(\cdot, \cdot), \sigma(\cdot, \cdot)$ are nonnegative functions belonging to $C\left(\mathbb{R}^{N} \times \mathbb{R}^{N}\right) \cap L^{\infty}\left(\mathbb{R}^{N} \times \mathbb{R}^{N}\right)$ and $\psi(x, \tilde{x})=\psi(\tilde{x}, x), \psi(x, x)=\sigma(x, x)=0$.

(H) $|a(x, \eta)-a(\tilde{x}, \tilde{\eta})| \leq \chi(x, \tilde{x})(|\eta|+|\tilde{\eta}|)+\mu(x, \tilde{x})|\eta-\tilde{\eta}|$, where $\chi$ has the same properties as $\psi$ and $\mu$ has the same properties as $\phi$.

Lemma 2. Suppose that the hypotheses $\left(\mathbf{H}_{1}\right)-\left(\mathbf{H}_{4}\right)$ take place. If $\psi \in C\left(\mathbb{R}^{N} \times \mathbb{R}^{N}\right) \cap$ $L^{\infty}\left(\mathbb{R}^{N} \times \mathbb{R}^{N}\right)$ and $S_{n}, S_{0} \in \mathcal{F}^{k, \infty}$ with $S_{n} \rightarrow S_{0}, S_{n}^{-1} \rightarrow S_{0}^{-1}$ in $W^{k, \infty}\left(\mathbb{R}^{N}\right)^{N}$, then

$$
\left\|\psi\left(S_{n}(\cdot), S_{0}(\cdot)\right)-\psi\left(S_{0}(\cdot), S_{0}(\cdot)\right)\right\|_{L^{\infty}(C)} \rightarrow 0
$$

when $n \rightarrow \infty$.

Proof. We have that $\psi$ is uniformly continuous on every bounded set of $\mathbb{R}^{N} \times \mathbb{R}^{N}$, which implies:

For each $\varepsilon>0$, there exists $\delta>0$ such that for each $x_{1}, \tilde{x}_{1}, x_{2}, \tilde{x}_{2} \in D$ (a bounded closed subset of $\mathbb{R}^{N}$ ), with $\left\|x_{1}-x_{2}\right\|_{N}<\delta$ and $\left\|\tilde{x}_{1}-\tilde{x}_{2}\right\|_{N}<\delta$ we have

$$
\begin{gathered}
\left|\psi\left(x_{1}, \tilde{x}_{1}\right)-\psi\left(x_{2}, \tilde{x}_{2}\right)\right|<\varepsilon . \\
S_{n} \rightarrow S_{0} \text { in } L^{\infty}(C) \text {, that is } \inf _{E \subset C,|E|=0} \sup _{X \in C \backslash E}\left\|S_{n}(X)-S_{0}(X)\right\|_{N} \rightarrow 0 .
\end{gathered}
$$

For $\delta>0$ there exists $n_{0} \in \mathbb{N}$ such that for $n>n_{0}$

$$
\inf _{E \subset C,|E|=0} \sup _{X \in C \backslash E}\left\|S_{n}(X)-S_{0}(X)\right\|_{N}<\delta .
$$

This implies that, for $n>n_{0}$, there exists a set $E_{n} \subset C,\left|E_{n}\right|=0$ such that $\left\|S_{n}(X)-S_{0}(X)\right\|_{N}<\delta$ for each $X \in C \backslash E_{n}$.

Using the uniform continuity and the fact that $S_{n}(C)$ and $S_{0}(C)$ are in a bounded set of $\mathbb{R}^{N}$, we get: There exists $n_{0} \in \mathbb{N}$ such that for all $n>n_{0}$, exists $E_{n} \subset C,\left|E_{n}\right|=0$ with

$$
\left|\psi\left(S_{n}(X), S_{0}(X)\right)-\psi\left(S_{0}(X), S_{0}(X)\right)\right|<\varepsilon, \forall X \in C \backslash E_{n}
$$


Denote $E=\cup_{n>n_{0}} E_{n},|E|=0, C \backslash E \subset C \backslash E_{n}$, so

$$
\begin{aligned}
& \sup _{X \in C \backslash E}\left|\psi\left(S_{n}(X), S_{0}(X)\right)-\psi\left(S_{0}(X), S_{0}(X)\right)\right| \\
\leq & \sup _{X \in C \backslash E_{n}}\left|\psi\left(S_{n}(X), S_{0}(X)\right)-\psi\left(S_{0}(X), S_{0}(X)\right)\right|<\varepsilon
\end{aligned}
$$

for all $n>n_{0}$. Finally, for $\varepsilon>0$ there exists $n_{0} \in \mathbb{N}$ such that for all $n>n_{0}$ we have

$$
\inf _{E \subset C,|E|=0} \sup _{X \in C \backslash E}\left|\psi\left(S_{n}(X), S_{0}(X)\right)-\psi\left(S_{0}(X), S_{0}(X)\right)\right| \leq \varepsilon,
$$

which completes the proof.

Suppose that the next hypothesis is satisfied in all the paper:

$\left(\mathbf{H}_{5}\right)$ There exists a neighborhood $V_{0}$ of $S_{0}$ and a positive constant $\delta$ such that for all $S_{1}, S_{2} \in V_{0}$ and $u_{1} \in K_{S_{1}}$, there exists $u_{2} \in K_{S_{2}}$ such that

$$
\left\|u_{1}-u_{2}\right\|_{H^{1}(C)} \leq \delta\left(\left\|S_{1}-S_{2}\right\|_{k, \infty}+\left\|S_{1}^{-1}-S_{2}^{-1}\right\|_{k, \infty}\right) .
$$

Example 1. Let $K(\Omega)=\left\{v \in H_{0}^{1}(\Omega) \mid \nabla v=0, v(x) \geq \varphi(x)\right.$ a.e. $\left.x \in \Omega\right\}$, with $\varphi \in H_{0}^{1}(\Omega) \cap C(\Omega)$ a Lipschitz function with $\nabla \varphi=0$ on $\Omega$.

We have:

$$
K_{S}=\{\check{v} \circ S \mid \check{v} \in K(\Omega)\}=\left\{v \in H_{0}^{1}(C) \mid \nabla v=0, v(X) \geq \varphi(S(X)) \text { a.e. } X \in C\right\}
$$

Let $S_{1}, S_{2} \in \mathcal{F}^{k, \infty}, u_{1} \in K_{S_{1}}$. This means $\nabla u_{1}=0$ and $u_{1} \geq \varphi\left(S_{1}(X)\right)$ a.e. on $C$. We define $u_{2}$ as:

$$
u_{2}(X)= \begin{cases}u_{1}(X), & \text { if } u_{1}(X) \geq \varphi\left(S_{2}(X)\right) \\ \varphi\left(S_{2}(X)\right), & \text { if } u_{1}(X)<\varphi\left(S_{2}(X)\right)\end{cases}
$$

We have $u_{2} \in K_{S_{2}}$. Obviously $\left|u_{1}(X)-u_{2}(X)\right| \leq\left|\varphi\left(S_{1}(X)\right)-\varphi\left(S_{2}(X)\right)\right|$. Moreover,

$$
\begin{aligned}
& \left\|u_{1}-u_{2}\right\|_{H^{1}(C)}^{2}=\int_{C}\left|u_{1}(X)-u_{2}(X)\right|^{2} d X \leq \int_{C}\left|\varphi\left(S_{1}(X)\right)-\varphi\left(S_{2}(X)\right)\right|^{2} d X \\
& \leq L^{2} \int_{C}\left|S_{1}(X)-S_{2}(X)\right|^{2} d X \leq L^{2}\left\|S_{1}-S_{2}\right\|_{k, \infty}^{2}|C|^{1 / 2}
\end{aligned}
$$

which shows that $\left(\mathbf{H}_{\mathbf{5}}\right)$ is satisfied.

Example 2. A special case when $\left(\mathbf{H}_{5}\right)$ is trivially satisfied is the case when $K=K_{S}$ is independent of $S \in \mathcal{F}^{k, \infty}$, for all $k \geq 1$. For example (see [4]), this happens for

$$
\begin{aligned}
& K(\Omega)_{1}=\left\{v \in H^{1}(\Omega)|v|_{\partial \Omega}=c, v \geq d \text { a.e. in } \Omega\right\} \\
& K(\Omega)_{2}=\left\{v \in H^{1}(\Omega) \mid v \geq f \text { a.e. in } \Omega\right\},
\end{aligned}
$$

where $c, d, f$ are constants. Then:

$$
\begin{aligned}
& S K(\Omega)_{1}=\left\{v \in H^{1}(C)|v|_{\partial C}=c, v \geq d \text { a.e. in } C\right\} \\
& S K(\Omega)_{2}=\left\{v \in H^{1}(C) \mid v \geq f \text { a.e. in } C\right\},
\end{aligned}
$$

which do not depend on $S$. 
We fix now an initial value $S_{0} \in \mathcal{F}^{k, \infty}, u_{0} \in K$ a solution of $(V I)_{S_{0}}$ and we prove the main theorem of the paper.

(In order to prove the main theorem, we suppose that:

$\left(\mathbf{H}_{\mathbf{6}}\right)\left|a_{j}(x, \xi)-a_{j}(x, \tilde{\xi})\right| \leq L_{1}\|\xi-\tilde{\xi}\|_{N}$ and $|a(x, \eta)-a(x, \tilde{\eta})| \leq L_{2}|\eta-\tilde{\eta}|$, a.e. $x \in \mathbb{R}^{N}$, for all $\xi, \tilde{\xi} \in \mathbb{R}^{N}$ and $\eta, \tilde{\eta} \in \mathbb{R}$, with $L_{1}, L_{2}$ positive constants.)

Theorem 3. Suppose that $\left(\mathbf{H}_{\mathbf{1}}\right)-\left(\mathbf{H}_{5}\right)$ are satisfied. Let $S_{0} \in \mathcal{F}^{k, \infty}$ and $u_{0} \in K_{S_{0}}$ fixed. If $u_{0}$ is a solution of the variational inequality $(V I)_{S_{0}}$, then $(V I)_{S_{0}}$ is stable under perturbations, that is: there exists a neighborhood $W_{0}$ of $S_{0}$ and a mapping $\theta: W_{0} \rightarrow H^{1}(C)$ such that for each $S \in W_{0}, \theta(S)=u_{S}$ is a solution of $(V I)_{S}$, $\theta\left(S_{0}\right)=u_{0}$ and $\theta$ is continuous in $S_{0}$.

Proof. We use Theorem 1 with $W:=\mathcal{F}^{k, \infty}, H=H^{1}(C), K: W \rightarrow 2^{H}, K(S)=K_{S}$ a set-valued map and $T: W \times H \rightarrow H^{*}, T(S, u)=\mathcal{A}(S, u)$. We check the hypotheses of Theorem 1.

(i) is obvious.

We prove now (iii), that is we show that there exists a neighborhood $V$ of $S_{0}$ such that the mappings $\mathcal{A}(S, \cdot)$ are uniformly strongly monotone for each $S \in V$.

We have:

$$
\begin{aligned}
& \langle\mathcal{A}(S, v)-\mathcal{A}(S, u), v-u\rangle \\
& =\int_{\Omega}\left[A\left(x, \nabla\left(v \circ S^{-1}\right)(x)\right)-A\left(x, \nabla\left(u \circ S^{-1}\right)(x)\right)\right] \cdot \nabla\left(v \circ S^{-1}-u \circ S^{-1}\right)(x) d x \\
& +\int_{\Omega}\left[a\left(x,\left(v \circ S^{-1}\right)(x)\right)-a\left(x,\left(u \circ S^{-1}\right)(x)\right)\right]\left(v \circ S^{-1}-u \circ S^{-1}\right)(x) d x \\
& \geq \int_{\Omega}\left\{\gamma_{1}\left\|\nabla\left(v \circ S^{-1}\right)(x)-\nabla\left(u \circ S^{-1}\right)(x)\right\|_{N}^{2}+\gamma_{2}\left|\left(v \circ S^{-1}\right)(x)-\left(u \circ S^{-1}\right)(x)\right|^{2}\right\} d x \\
& \geq \gamma\left\|v \circ S^{-1}-u \circ S^{-1}\right\|_{H^{1}(\Omega)} \geq \frac{\gamma}{\left\|\operatorname{det} J S^{-1}\right\|_{\infty}\left(1+\|J S\|_{\infty}\right)^{2}}\|v-u\|_{H^{1}(C)}^{2} \\
& \geq \tilde{\gamma}\|v-u\|_{H^{1}(C)}^{2} .
\end{aligned}
$$

In this evaluation we made use of the transform $X=S^{-1}(x)$, the hypothesis (P4) and of the continuity of the mappings $S \mapsto J S$ and $S \mapsto \operatorname{det} J S^{-1}$ (see Lemma 1, c).

Next the continuity of $\mathcal{A}(S, \cdot)$ from $H^{1}(C)$ with the strong topology to $\left(H^{1}(C)\right)^{*}$ with the weak topology will be proved.

Let $u_{n} \rightarrow u$ in $H^{1}(C)$ and let $v \in H^{1}(C)$. We get:

$$
\begin{aligned}
& \left|\left\langle\mathcal{A}\left(S, u_{n}\right), v\right\rangle-\langle\mathcal{A}(S, u), v\rangle\right| \\
& \leq\|\operatorname{det} J S\|_{\infty}\left\{\|\phi(S(\cdot), S(\cdot))\|_{\infty}\left\|J S^{-t}\right\|_{\infty}^{2}\right. \\
& \left.+\|\mu(S(\cdot), S(\cdot))\|_{\infty}\right\}\left\|u_{n}-u\right\|_{H^{1}(C)}\|v\|_{H^{1}(C)} \rightarrow 0 .
\end{aligned}
$$

There is still to be proved (ii) from Theorem 1, that is the consistency of $T$. 
Let $0<r \leq 1$. We consider $S \in V_{0}$ and in $\left(\mathbf{H}_{5}\right)$ we put $S_{1}:=S_{0}, S_{2}:=S$, $u_{1}:=u_{0}$. Then there exists $u_{S} \in K_{S}$ such that

$$
\left\|u_{S}-u_{0}\right\|_{H^{1}(C)} \leq \delta\left(\left\|S-S_{0}\right\|_{k, \infty}+\left\|S^{-1}-S_{0}^{-1}\right\|_{k, \infty}\right) .
$$

We define

$$
\beta(S)=\max \left\{\sqrt{\delta\left(\left\|S-S_{0}\right\|+\left\|S^{-1}-S_{0}^{-1}\right\|\right)}, 2\left\|\mathcal{A}\left(S, u_{S}\right)-\mathcal{A}\left(S_{0}, u_{0}\right)\right\|_{\left(H^{1}(C)\right)^{*}}\right\} .
$$

Obviously $\beta\left(S_{0}\right)=0$ (we take $u_{S_{0}}=u_{0}$ ). We prove next that $\beta$ is continuous at $S_{0}$.

For $\sqrt{\delta\left(\left\|S-S_{0}\right\|+\left\|S^{-1}-S_{0}^{-1}\right\|\right)}$ this is obvious.

For the second term, we consider $S_{n} \rightarrow S_{0}$ in $\mathcal{F}^{k, \infty}$. Then, according to $\left(\mathbf{H}_{5}\right)$, $u_{S_{n}} \rightarrow u_{0}$ in $H^{1}(C)$.

We evaluate $\left|\left\langle\mathcal{A}\left(S_{n}, u_{S_{n}}\right)-\mathcal{A}\left(S_{0}, u_{0}\right), v\right\rangle\right|$ for $v \in H^{1}(C)$.

We have

$$
\left|\left\langle\mathcal{A}\left(S_{n}, u_{S_{n}}\right)-\mathcal{A}\left(S_{0}, u_{S_{n}}\right), v\right\rangle\right| \leq\left(\alpha_{1 n}+\alpha_{2 n}+\alpha_{3 n}+\alpha_{4 n}\right)\|v\|_{H^{1}(C)}
$$

where

$$
\begin{aligned}
& \alpha_{1 n}=\left\|\operatorname{det} J S_{n}\right\|_{\infty} N\left\{\left\|\psi\left(S_{n}(\cdot), S_{0}(\cdot)\right)\right\|_{\infty}\left\|J S_{n}^{-t}\right\|_{\infty}\left(\left\|J S_{n}^{-t}\right\|_{\infty}+\left\|J S_{0}^{-t}\right\|_{\infty}\right)\right. \\
& \left.+\left\|\phi\left(S_{n}(\cdot), S_{0}(\cdot)\right)\right\|_{\infty}\left\|J S_{n}^{-t}\right\|_{\infty}\left\|J S_{n}^{-t}-J S_{0}^{-t}\right\|_{\infty}\right\}\left\|u_{S_{n}}\right\|_{H^{1}(C)} \\
& +\left\|\operatorname{det} J S_{n}\right\|_{\infty} N\left\|\sigma\left(S_{n}(\cdot), S_{0}(\cdot)\right)\right\|_{\infty}|C|^{1 / 2}\left\|J S_{n}^{-t}\right\|_{\infty} \\
& +\left\|\operatorname{det} J S_{n}\right\|_{\infty}\left\|J S_{n}^{-t}-J S_{0}^{-t}\right\|_{\infty} c N\left\{\left(\int_{C}\left|k\left(S_{0}(X)\right)\right|^{2} d X\right)^{1 / 2}+\left\|J S_{0}^{-t}\right\|_{\infty}\right\}\left\|u_{S_{n}}\right\|_{H^{1}(C)}, \\
& \alpha_{2 n}=\left\|\operatorname{det} J S_{n}-\operatorname{det} J S_{0}\right\|_{\infty}\left\|J S_{0}^{-t}\right\|_{\infty} c N\left\{\left(\int_{C}\left|k\left(S_{0}(X)\right)\right|^{2} d X\right)^{1 / 2}\right. \\
& \left.+\left\|J S_{0}^{-t}\right\|_{\infty}\left\|u_{S_{n}}\right\|_{H^{1}(C)}\right\}, \\
& \alpha_{3 n}=\left\|\operatorname{det} J S_{n}\right\|_{\infty}\left\|\chi\left(S_{n}(\cdot), S_{0}(\cdot)\right)\right\|_{\infty} 2\left\|u_{S_{n}}\right\|_{H^{1}(C)}, \\
& \alpha_{4 n}=\left\|\operatorname{det} J S_{n}-\operatorname{det} J S_{0}\right\|_{\infty} c_{1}\left\{\left(\int_{C}\left|k_{1}\left(S_{0}(X)\right)\right|^{2} d X\right)^{1 / 2}+\left\|u_{S_{n}}\right\|_{H^{1}(C)}\right\}
\end{aligned}
$$

Next, as in the proof of (iii), one can obtain

$$
\left|\left\langle\mathcal{A}\left(S_{0}, u_{S_{n}}\right)-\mathcal{A}\left(S_{0}, u_{0}\right), v\right\rangle\right| \leq \alpha_{5 n}\|v\|_{H^{1}(C)}
$$

where

$$
\alpha_{5 n}=\left\|\operatorname{det} J S_{0}\right\|_{\infty}\left\{\left\|\phi\left(S_{0}(\cdot), S_{0}(\cdot)\right)\right\|_{\infty}\left\|J S_{0}^{-t}\right\|_{\infty}^{2}+\left\|\mu\left(S_{0}(\cdot), S_{0}(\cdot)\right)\right\|_{\infty}\right\}\left\|u_{S_{n}}-u_{0}\right\|_{H^{1}}
$$


From the hypotheses imposed it is clear that $\alpha_{n}=\sum_{i=1}^{5} \alpha_{i n} \rightarrow 0$ when $n \rightarrow 0$. So we get:

$$
\begin{aligned}
& \left\|\mathcal{A}\left(S_{n}, u_{S_{n}}\right)-\mathcal{A}\left(S_{0}, u_{0}\right)\right\|_{\left(H^{1}(C)\right)^{*}} \\
& =\sup \left\{\left|\left\langle\mathcal{A}\left(S_{n}, u_{S_{n}}\right)-\mathcal{A}\left(S_{0}, u_{0}\right), v\right\rangle\right|: v \in H^{1}(C),\|v\|_{H^{1}(C)} \leq 1\right\} \\
& \leq \sup \left\{\alpha_{n}\|v\|_{H^{1}(C)}: v \in H^{1}(C),\|v\|_{H^{1}(C)} \leq 1\right\} \leq \alpha_{n} \rightarrow 0 .
\end{aligned}
$$

From this the continuity of $\beta$ at $S_{0}$ is clear.

Let $W_{r} \subset V_{0}$ be a neighbourhood of $S_{0}$ such that for each $S \in W_{r}$

$$
\beta(S) \leq 1 \quad \text { and } \quad r-4 \beta(S)\left\|\mathcal{A}\left(S_{0}, u_{0}\right)\right\|_{\left(H^{1}(C)\right)^{*}} \geq 0 .
$$

Let $v \in K_{S}$ with $r \leq\left\|v-u_{S}\right\|$. We have:

$$
\begin{aligned}
\left\langle\mathcal{A}\left(S, u_{S}\right), v-u_{S}\right\rangle+\beta(S)\left\|v-u_{S}\right\| \\
=\left\langle\mathcal{A}\left(S, u_{S}\right)-\mathcal{A}\left(S_{0}, u_{0}\right), v-u_{S}\right\rangle+\left\langle\mathcal{A}\left(S_{0}, u_{0}\right), v-u_{S}\right\rangle+\beta(S)\left\|v-u_{S}\right\| \\
\geq-\left\|\mathcal{A}\left(S, u_{S}\right)-\mathcal{A}\left(S_{0}, u_{0}\right)\right\|\left\|v-u_{S}\right\|+\left\langle\mathcal{A}\left(S_{0}, u_{0}\right), v-u_{S}\right\rangle+\beta(S)\left\|v-u_{S}\right\| \\
\geq-\frac{1}{2} \beta(S)\left\|v-u_{S}\right\|+\beta(S)\left\|v-u_{S}\right\|+\left\langle\mathcal{A}\left(S_{0}, u_{0}\right), v-u_{0}\right\rangle+\left\langle\mathcal{A}\left(S_{0}, u_{0}\right), u_{0}-u_{S}\right\rangle \\
\geq \frac{1}{2} \beta(S)\left\|v-u_{S}\right\|+\left\langle\mathcal{A}\left(S_{0}, u_{0}\right), v-v_{0}\right\rangle+\left\langle\mathcal{A}\left(S_{0}, u_{0}\right), v_{0}-u_{0}\right\rangle \\
\quad-\left\|\mathcal{A}\left(S_{0}, u_{0}\right)\right\|\left\|u_{0}-u_{S}\right\| \\
\geq \frac{1}{2} \beta(S)\left\|v-u_{S}\right\|-\left\|v-v_{0}\right\|\left\|\mathcal{A}\left(S_{0}, u_{0}\right)\right\|-\left\|u_{0}-u_{S}\right\|\left\|\mathcal{A}\left(S_{0}, u_{0}\right)\right\| \\
\geq \frac{1}{2} \beta(S)\left[r-4 \beta(S)\left\|\mathcal{A}\left(S_{0}, u_{0}\right)\right\|\right] \geq 0 .
\end{aligned}
$$

(Here we considered $v_{0} \in K_{S_{0}}$ such that $\left\|v-v_{0}\right\| \leq \delta\left(\left\|S-S_{0}\right\|+\left\|S^{-1}-S_{0}^{-1}\right\|\right)$ ).

On the other hand we have $\left\|u_{S}-u_{0}\right\| \leq \beta^{2}(S) \leq \beta(S)$, which concludes the proof.

Example 3. We present a linear variational inequality for which all the previous hypotheses are satisfied. Consider the problem:

Find $u_{\Omega} \in K(\Omega)$ such that $a_{\Omega}\left(u_{\Omega}, v-u_{\Omega}\right) \geq 0, \forall v \in K(\Omega)$,

where

$$
a_{\Omega}(u, v)=\int_{\Omega}[B(x) \nabla u(x) \cdot \nabla v(x)+b(x) u(x) v(x)] d x
$$

with $B \in C\left(\mathbb{R}^{N}\right)^{N^{2}} \cap L^{\infty}\left(\mathbb{R}^{N}\right)^{N^{2}}, b \in C\left(\mathbb{R}^{N}\right) \cap L^{\infty}\left(\mathbb{R}^{N}\right), b(x) \geq \tilde{b}>0$ and

$$
\sum_{j=1}^{N} \sum_{k=1}^{N} b_{j k}(x) \xi_{k} \xi_{j} \geq \alpha\|\xi\|_{N}^{2}
$$


a.e. $x \in \mathbb{R}^{N}$, for all $\xi \in \mathbb{R}^{N}$. It can be easily shown that, with

$$
a_{j}(x, \xi)=\sum_{k=1}^{N} b_{j k}(x) \xi_{k}=B_{j}(x) \cdot \xi \text { and } a(x, \eta)=b(x) \eta
$$

the hypotheses $\left(\mathbf{H}_{\mathbf{1}}\right)-\left(\mathbf{H}_{\mathbf{4}}\right)$ are satisfied.

\section{REFERENCES}

[1] Soкolowsкi, J. and Zolesio, J.P.: Introduction to Shape Optimization. Shape Sensitivity Analysis, Springer-Verlag, 1992.

[2] Pironneau, O.: Optimal Shape Design for Elliptic Systems, Springer-Verlag, New-York, 1984.

[3] Murat, F. and Simon, J.: Sur le Controle par un Domaine Geometrique, Preprint No. 76015, University of Paris, 1976.

[4] Liu W. B. and RuBio J. E.: Optimal shape design for systems governed by variational inequalities, Part 1: Existence theory for elliptic case, Part 2: Existence theory for evolution case, J. of Optimization Theory and Applications, 69, (1991), 351-371, 373-396.

[5] Denkowski, Z. and Migórski S.: Optimal shape design problems for a class of systems described by hemivariational inequalities, J. of Global Optimization, 12, (1998), 37-59.

[6] Gasinski, L.: Mapping Method in Optimal Shape Design Problems Governed by Hemivariational Inequalities, Shape Optimization and Optimal Design (edited by J. Cagnol, M.P. Polis, J.P. Zolesio), Dekker, New York, (2001), 277-288.

[7] Kassay, G. and Kolumbán, J.: Multivalued Parametric Variational Inequalities with $\alpha$ Pseudomonotone Maps, J. of Optimization Theory and Applications, 107, No.1 (2000), 35-50.

[8] Atr, W. and Kolumbán, I.: An implicit function theorem for a class of monotone generalized equations, Kybernetica, 29, (1993), 210-221.

[9] Showalter, R. E.: Monotone Operators in Banach Space and Nonlinear Partial Differential Equations, American Mathematical Society, Mathematical Surveys and Monographs, 49, 1997.

\section{Author's Address}

\section{Daniela Inoan:}

Department of Mathematics, Technical University of Cluj-Napoca, 15, C. Daicoviciu str., 3400 Cluj, Romania

E-mail address: Daniela. Inoan@math.utcluj.ro 